\section{Aumento no uso de transporte motorizado privado no deslocamento das crianças para a escola na Região Metropolitana de São Paulo, Brasil, 1997-2012}

\author{
Use of private motor vehicle transportation for \\ taking children to school in São Paulo Metropolitan \\ Area, Brazil, 1997-2012
}

\begin{abstract}
Aumento en el uso de transporte motorizado privado en el desplazamiento de los niños hacia la escuela en la Región Metropolitana de São Paulo, Brasil, 1997-2012
\end{abstract}

\begin{abstract}
Resumo
A Região Metropolitana de São Paulo, Brasil, apresentou, entre 1997 e 2007, tendência de aumento do uso transporte motorizado privado nos deslocamentos de crianças para a escola, com potenciais prejuízos à saúde. O objetivo deste estudo foi ampliar a análise de tal tendência para 2012 e discutir possíveis estratégias para aumentar a proporção de crianças que andam, pedalam e usam o transporte público. A análise dos dados da Pesquisa de Mobilidade de 2012 indica não apenas a continuidade, mas a aceleração no aumento do uso transporte motorizado privado em deslocamentos de crianças entre 6 e 11 anos para a escola. O efeito de iniciativas em andamento sobre essa tendência só será devidamente compreendido com o devido monitoramento dos deslocamentos cotidianos e a avaliação do impacto dessas ações sobre a saúde da população. Um pacote de políticas e programas voltados especificamente para a promoção e proteção da mobilidade a pé, de bicicleta ou por transporte público de crianças é imprescindivel para garantir o deslocamento seguro, independente e ativo de crianças para a escola na Região Metropolitana de São Paulo.
\end{abstract}

Caminhada; Transportes; Criança
Thiago Hérick de Sá 1

Leandro Fórnias Machado de Rezende 2 Fabiana Maluf Rabacow 3 Carlos Augusto Monteiro 1

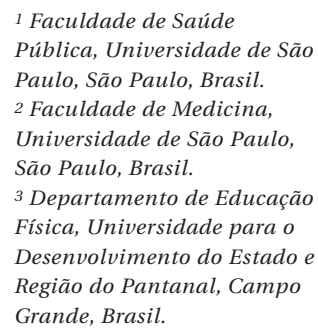

1 Faculdade de Saúde Pública, Universidade de São Paulo, São Paulo, Brasil. 2 Faculdade de Medicina, Universidade de São Paulo, São Paulo, Brasil.

3 Departamento de Educação Física, Universidade para o Desenvolvimento do Estado e Região do Pantanal, Campo Grande, Brasil.

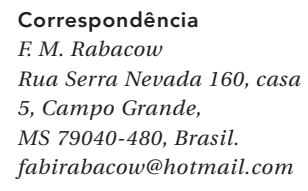




\section{Introdução}

De acordo com os dados coletados em 1997 e 2007 pela Pesquisa Origem e Destino conduzida na Região Metropolitana de São Paulo, Brasil, aproximadamente $12 \%$ das crianças entre 6 e 11 anos trocaram a caminhada, a bicicleta e o transporte público pelo transporte motorizado privado nos seus deslocamentos para a escola 1. Formas mais ativas de deslocamento trazem reconhecidos benefícios à saúde do indivíduo e da população ${ }^{2}$, sendo apontadas como um dos caminhos imprescindíveis para combater alguns dos maiores desafios atuais da saúde pública, como a pandemia de doenças crônicas não transmissíveis e as mudanças climáticas 3 . A opção pelo transporte público ou pela caminhada ou bicicleta para deslocamentos também traz importantes cobenefícios, como a redução da poluição atmosférica e acidentes de trânsito 2,3 .

Além disso, o uso do transporte público, da caminhada ou da bicicleta para ir à escola implica fortalecimento da capacidade de as crianças se moverem de forma independente em sua cidade ${ }^{4}$. Tal condição é fundamental na sua formação como cidadão. É também um indicativo da percepção dos pais sobre os riscos fora de casa aos quais suas crianças estão submetidas ${ }^{5}$. Mais ainda, garantir a participação de crianças nas vias e no sistema público de transporte favorece a diversidade e reforça o papel dos espaços públicos como condutores da saúde e da prosperidade das cidades 6 .

A realização de nova coleta de dados pela Pesquisa de Mobilidade em 2012 permite estabelecer a variação recente dos modos de transporte para a escola utilizados pelas crianças da Região Metropolitana de São Paulo. O objetivo desta comunicação é descrever a variação na proporção de crianças que andam, pedalam e usam o transporte público nos seus deslocamentos para a escola.

\section{Métodos}

Embora o número de domicílios e de pessoas estudados na Pesquisa de Mobilidade de 2012 tenha sido inferior ao das pesquisas anteriores (Tabela 1), o processo de amostragem foi semelhante, sendo as unidades primárias de amostragem (zonas) de 2012 construídas como conglomerados das zonas de 2007, de modo que os limites territoriais fossem respeitados e que o processo de zoneamento cobrisse todo o território dos 39 municípios da Região Metropolitana de São Paulo (Tabela 1). As estimativas para 2012 foram calculadas de modo idêntico ao empregado nas estimativas relativas aos dois anos anteriores 1 . Foram consideradas como diferenças estatisticamente significantes proporções cujas estimativas pontuais não estivessem contidas no intervalo de 95\% de confiança (IC95\%) da proporção a ser comparada 7 e como populacionalmente relevantes diferenças de magnitude superior a dois pontos percentuais.

\section{Resultados e Discussão}

A análise dos dados da Pesquisa de Mobilidade de 2012 indica não apenas continuidade, mas aceleração da queda no número de crianças que andam, pedalam e usam o transporte público na Região Metropolitana de São Paulo (Figuras 1a e 1b). A mesma redução de aproximadamente dez pontos percentuais na proporção de crianças que se deslocam ativamente observada entre 1997 e 2007 (intervalo de dez anos) também foi encontrada entre 2007 e 2012 (intervalo de cinco anos), tanto em meninos quanto em meninas (Tabela 2). Em havendo continuidade dessa tendência, até 2017, ano da próxima Pesquisa Origem e Destino, a proporção de crianças que vão à escola por modos motorizados privados ultrapassará 50\%. Entre os adolescentes (12 a 17 anos), a proporção de deslocamentos por modo de transporte permanece estável no período, com aproximadamente $67 \%$ dos adolescentes deslocando-se ativamente, $17 \%$ por transporte público e $15 \%$ por transporte motorizado privado, em meninos e meninas.

Apesar de existirem diferenças metodológicas entre as Pesquisa Origem e Destino de 1997 e 2007 e a Pesquisa de Mobilidade de 2012, esses levantamentos nos parecem suficientemente comparáveis e capazes de prover estimativas do padrão de deslocamento da população da Região Metropolitana de São Paulo. Entretanto, o grande intervalo entre essas pesquisas, em contraponto à urgência de medidas para reverter essa tendência negativa, ilustra a necessidade de um sistema de monitoramento detalhado sobre o padrão de deslocamentos da população. Ainda que muito úteis para estudos em saúde pública, a Pesquisa Origem e Destino têm propósito original distinto, e apenas um planejamento multissetorial aprimoraria a coleta e uso das informações sobre mobilidade urbana e seus possíveis impactos na saúde pública.

A piora verificada na tendência também evidencia a necessidade de ações que estimulem o uso da caminhada, da bicicleta ou do transporte público para o deslocamento de crianças e adolescentes. Algumas iniciativas na região 
Tabela 1

Detalhes sobre as pesquisas utilizadas para a avaliação da tendência de caminhada e uso da bicicleta nos deslocamentos para a escola. Região Metropolitana de São Paulo, Brasil, 1997-2012.

\begin{tabular}{|c|c|c|c|}
\hline & 1997 & 2007 & 2012 \\
\hline Nome da pesquisa & Pesquisa Origem e Destino & Pesquisa Origem e Destino & Pesquisa de Mobilidade \\
\hline Realização & $\begin{array}{l}\text { Companhia do Metropolitano de } \\
\text { São Paulo/Secretaria de Transportes } \\
\text { Metropolitanos do Estado de } \\
\text { São Paulo/Banco Nacional de } \\
\text { Desenvolvimento Econômico e Social }\end{array}$ & $\begin{array}{l}\text { Companhia do Metropolitano de } \\
\text { São Paulo/Secretaria de Transportes } \\
\text { Metropolitanos do Estado de São } \\
\text { Paulo }\end{array}$ & $\begin{array}{l}\text { Companhia do Metropolitano de } \\
\text { São Paulo/Secretaria de Transportes } \\
\text { Metropolitanos do Estado de São Paulo }\end{array}$ \\
\hline População estimada & 16.859 .712 & 19.223 .930 & 19.956 .590 \\
\hline Amostragem & $\begin{array}{c}\text { Amostragem de domicílios, por } \\
\text { conglomerado (zonas), estratificada } \\
\text { pelo consumo domiciliar de energia } \\
\text { elétrica, com reposição }\end{array}$ & $\begin{array}{c}\text { Amostragem de domicílios, por } \\
\text { conglomerado (zonas), estratificada } \\
\text { pelo consumo domiciliar de energia } \\
\text { elétrica, com reposição }\end{array}$ & $\begin{array}{l}\text { Amostragem de domicílios, por } \\
\text { conglomerado (zonas), estratificada pelo } \\
\text { consumo domiciliar de energia elétrica, } \\
\text { com reposição }\end{array}$ \\
\hline Total de zonas & 389 & 460 & 31 \\
\hline $\begin{array}{l}\text { Amostra total } \\
\text { (domicílios) }\end{array}$ & $98.780(26.278)$ & 91.405 (29.957) & $24.534(8.115)$ \\
\hline $\begin{array}{l}\text { Total de crianças (6 a } \\
11 \text { anos) }\end{array}$ & 7.604 & 5.795 & 1.628 \\
\hline Período de coleta & 1997 & $\begin{array}{l}\text { Agosto a novembro de } 2007 \text { e } \\
\text { fevereiro a abril de } 2008\end{array}$ & 2012 \\
\hline Estratégia de coleta & $\begin{array}{l}\text { Entrevistas domiciliares: questionário } \\
\text { sociodemográfico + diário das viagens } \\
\text { do dia anterior }\end{array}$ & $\begin{array}{l}\text { Entrevistas domiciliares: questionário } \\
\text { sociodemográfico + diário das viagens } \\
\text { do dia anterior }\end{array}$ & $\begin{array}{c}\text { Entrevistas domiciliares: questionário } \\
\text { sociodemográfico + diário das viagens } \\
\text { do dia anterior }\end{array}$ \\
\hline
\end{tabular}

Figura 1

Tendência temporal dos modos de transporte utilizados por crianças de 6 a 11 anos nos deslocamentos para a escola. Região Metropolitana de São Paulo, Brasil, 1997-2012.

1a) Meninos

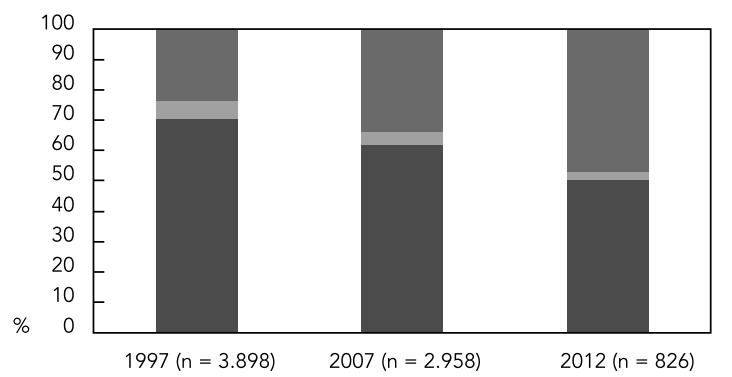

1b) Meninas

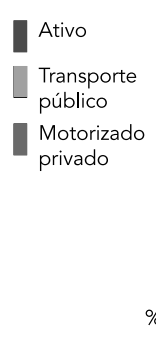

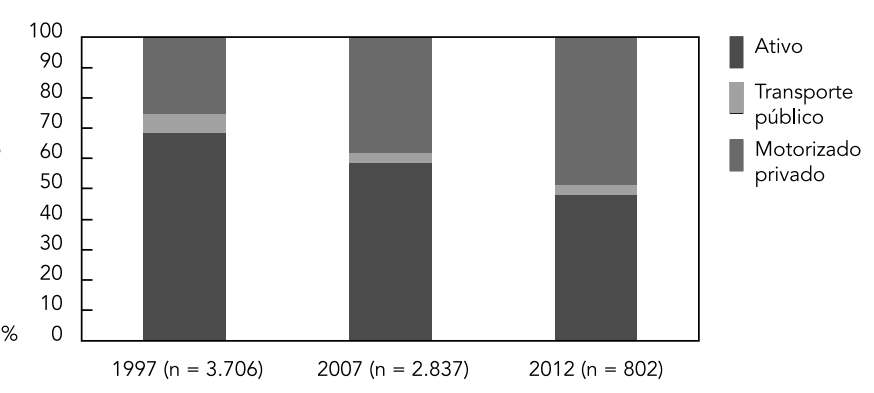


Distribuição dos modos de transporte utilizados por crianças de 6 a 11 anos nos deslocamentos para a escola. Região Metropolitana de São Paulo, Brasil, 1997-2012.

\begin{tabular}{|c|c|c|c|c|c|c|}
\hline \multirow[t]{2}{*}{ Meninos } & \multicolumn{2}{|c|}{$\begin{array}{c}1997 \\
(n=3.898)\end{array}$} & \multicolumn{2}{|c|}{$\begin{array}{c}2007 \\
(n=2.958)\end{array}$} & \multicolumn{2}{|c|}{$\begin{array}{c}2012 \\
(n=826)\end{array}$} \\
\hline & $\%$ & IC95\% & $\%$ & IC95\% & $\%$ & IC95\% \\
\hline Ativo & 70,5 & $68,2-72,8$ & 61,7 & $59,1-64,3$ & 50,0 & $46,2-53,9$ \\
\hline Transporte público & 5,8 & $4,8-6,8$ & 4,4 & $3,2-5,5$ & 3,0 & $1,7-4,3$ \\
\hline Motorizado privado & 23,7 & $21,4-25,9$ & 33,9 & $31,4-36,4$ & 46,9 & $43,0-50,8$ \\
\hline \multirow[t]{2}{*}{ Meninas } & \multicolumn{2}{|c|}{$\begin{array}{c}1997 \\
(n=3.706)\end{array}$} & \multicolumn{2}{|c|}{$\begin{array}{c}2007 \\
(n=2.837)\end{array}$} & \multicolumn{2}{|c|}{$\begin{array}{c}2012 \\
(n=802)\end{array}$} \\
\hline & $\%$ & IC95\% & $\%$ & IC95\% & $\%$ & IC95\% \\
\hline Ativo & 68,5 & $66,2-70,8$ & 58,7 & $56,0-61,3$ & 48,2 & $44,2-52,1$ \\
\hline Transporte público & 6,1 & $5,1-7,2$ & 3,2 & $2,3-4,2$ & 2,9 & $1,5-4,3$ \\
\hline Motorizado privado & 25,3 & $23,1-27,5$ & 38,1 & $35,5-40,7$ & 48,9 & $45,0-52,9$ \\
\hline
\end{tabular}

caminham nesta direção, como a isenção da tarifa para estudantes de escolas públicas nos serviços de transporte público estadual e de alguns municípios da Região Metropolitana de São Paulo, desde 2014. Em Londres (Reino Unido), a isenção da tarifa para qualquer pessoa menor de 17 anos, implantada em 2005, contribuiu para aumentar em $26 \%$ o uso de transporte público entre os mais jovens, sem que tenha havido redução nos deslocamentos ativos 8,9. A isenção da tarifa também normalizou o uso do transporte público e transformou a viagem de ônibus em importante espaço público de interação social para esse grupo populacional 8,9 .

Outras ações têm o potencial de reverter a tendência encontrada na Região Metropolitana de São Paulo, como a revisão do Plano Diretor Estratégico do Município de São Paulo e a elaboração dos Planos Municipais de Mobilidade Urbana, que têm como um dos princípios fundamentais a priorização do pedestre, do ciclista e do usuário de transporte público. Na contramão de tais iniciativas, programas federais largamente responsáveis pelo aumento da motorização na região, como políticas de subsídio a veículos motorizados e programas habitacionais que reforçam a segregação espacial da cidade, seguem em curso. O real impacto dessas iniciativas na motorização dos deslocamentos para a escola na Região Metropolitana de São Paulo somente será melhor compreendido apenas com o adequado monitoramento e avaliação do impacto dessas políticas à saúde pública 10 .
Paralelamente aos planos citados, também é necessário um pacote de políticas e programas voltados especificamente para promoção e proteção da mobilidade a pé, de bicicleta ou por transporte público de crianças e adolescentes 10. A própria escola e a comunidade podem servir como elemento propulsor e lócus destas iniciativas, dada a evidência de efetividade da promoção de modos de vida ativos a partir do ambiente escolar em regiões latino-americanas 11. Um bom exemplo de programa nacional consolidado de promoção da caminhada e do uso da bicicleta para a escola a partir do ambiente escolar é o Safe Routes to School (SRTS), iniciativa que envolve sociedade civil, escolas e governos, e que busca promover a saúde e qualidade de vida de crianças por meio do incentivo à caminhada ou uso de bicicleta para o deslocamento para (escola (nos Estados Unidos, desde 2005). Entre diversas ações, o SRTS examina as condições no entorno da escola e desenvolve ações para melhorar acessibilidade e segurança, reduzir trânsito e a poluição e fortalecer o suporte comunitário aos deslocamentos ativos infantis. O SRTS apresenta evidências de efetividade no aumento da segurança 12 e da caminhada ou uso de bicicleta para o deslocamento para a escola 13 .

Os efeitos de longo prazo desse conjunto de ações podem ser constatados no Japão, país que desenvolve uma "cultura de caminhada à escola" pelo menos desde 1953 14. Algumas iniciativas se destacam, como a proibição da circulação de carros em zonas escolares durante o horário de 
entrada e saída de alunos, a forte participação comunitária e a educação das crianças desde o jardim de infância sobre noções de trânsito. Não por acaso, há raros deslocamentos para escola por modo motorizado (modos privados ou transporte público). A imensa maioria das crianças caminha, inclusive aqueles menores de oito anos de idade, sem o acompanhamento dos pais 14 .

Exemplos como os citados acima devem ser adaptados à condição da Região Metropolitana de São Paulo e implementados para atenuar e futuramente reverter a tendência de motorização dos deslocamentos infantis. Para tanto, são fundamentais políticas integradas e ações multissetoriais, além da revisão e ampliação de programas já existentes, como o Transporte Escolar Gratuito, a fim de garantir o deslocamento seguro, independente e ativo de crianças para a escola.

\section{Conclusão}

No Brasil, é comum ouvir-se o discurso conservador de que "é preciso tirar as crianças das ruas". O entendimento, aqui, é absolutamente distinto. A construção de uma cidade mais humana, justa e sustentável ${ }^{3}$ passa por devolver não apenas as crianças às ruas, mas também seus pais, avós, amigos e vizinhos. São nas ruas que muitas das relações sociais e culturais acontecem e o deslocamento para a escola representa uma importante prática cotidiana da vida comunitária, com potencial para reinserção das crianças ao espaço público e à coletividade 10 .

\section{Colaboradores}

T. H. Sá foi responsável pela concepção e análises e colaborou na redação do texto. L. F. M. Rezende, F. M. Rabacow e C. A. Monteiro contribuíram com a revisão das análises e colaboraram na redação do texto.

\section{Agradecimentos}

T. H. Sá e L. F. M. Rezende gostariam de agradecer o apoio financeiro da Fundação de Amparo à Pesquisa de São Paulo (FAPESP, processos no 2012/08565-4 e no 2014/25614-4) para a realização desta pesquisa. 


\section{Referências}

1. Sá TH, Garcia LMT, Mielke GI, Rabacow FM, Rezende LFM. Changes in travel to school patterns among children and adolescents in the São Paulo Metropolitan Area, 1997-2007. J Transp Health 2015; 2:143-50.

2. Nazelle A, Nieuwenhuijsen MJ, Anto JM, Brauer M, Briggs D, Braun-Fahrlander C, et al. Improving health through policies that promote active travel: a review of evidence to support integrated health impact assessment. Environ Int 2011; 37:766-77.

3. Whitmee S, Haines A, Beyrer C, Boltz F, Capon AG, Souza Dias BF, et al. Safeguarding human health in the Anthropocene epoch: report of The Rockefeller-Foundation-Lancet-Commission on planetary health. Lancet 2015; 386:1973-2028.

4. Tranter P, Whitelegg J. Children's travel behaviours in Canberra: car-dependent lifestyles in a lowdensity city. J Transp Geogr 1994; 2:265-73.

5. Shaw B, Watson B, Frauendienst B, Redecker A, Jones T, Hullman M. Children's independent mobility: comparative study in England and Germany (1971-2010). London: Policy Studies Institute; 2013.

6. UN-Habitat. Streets as public spaces and drivers of urban prosperity. Nariobi: UN-Habitat; 2013.

7. Krzywinski M, Altman N. Points of significance: error bars. Nat Methods 2013; 10:921-2.
8. Edwards P, Steinbach R, Green J, Petticrew M, Goodman A, Jones A, et al. Health impacts of free bus travel for young people: evaluation of natural experiment in London. J Epidemiol Community Health 2013; 67:641-7.

9. Jones A, Steinbach R, Roberts H, Goodman A, Green J. Rethinking passive transport: bus fare exemptions and young people's wellbeing. Health Place 2012; 18:605-12.

10. Bruun E, Givoni M. Sustainable mobility: six research routes to steer transport policy. Nature 2015; 52329-31.

11. Hoehner CM, Ribeiro IC, Parra DC, Reis RC, Azevedo MR, Hino AA, et al. Physical activity interventions in Latin America: expanding and classifying the evidence. Am J Prev Med 2013; 44:e31-40.

12. Dimaggio C, Li G. Effectiveness of safe routes to school program in preventing school-aged pedestrian injury. Pediatrics 2013; 131:290-6.

13. Stewart O, Moudon AV, Claybrooke C. Multistate evaluation of safe routes to school programs. Am J Health Promot 2014; 28(3 Suppl):S89-96.

14. Mori N, Armada F, Willcox DC. Walking to school in Japan and childhood obesity prevention: new lessons from an old policy. Am J Public Health 2012; 102:2068-73. 


\section{Abstract}

São Paulo Metropolitan Area, Brazil, showed an in crease from 1997 to 2007 in the use of private motor vehicles for taking children to school, with potential harm to their health. The aim of this study was to extend the analysis of this trend until 2012 and discuss possible strategies to increase the proportion of children who walk, cycle, or use public transportation to get to school. Analysis of the data from the Mobility Survey of 2012 indicate not only the continuation but also an accelerated increase in the use of private motorized transportation for schoolchildren aged 6 to 11 years. The effect of initiatives to promote walking will only be properly understood with adequate monitoring of daily commuting to school and the evaluation of their impact on the population's health. A package of policies and programs specifically targeted to the promotion and protection of walking, cycling, and use of public transport by schoolchildren is indispensable for guaranteeing their right to travel safely, independently, and actively in São Paulo Metropolitan Area.

Walking; Transportation; Child

\section{Resumen}

La Región Metropolitana de São Paulo, Brasil, presentó, entre 1997 y 2007, una tendencia de aumento del uso transporte motorizado privado en los desplazamientos de niños hacia la escuela, con potenciales prejuicios para la salud. El objetivo de este estudio fue ampliar el análisis de tal tendencia en 2012, y discutir posibles estrategias para aumentar la proporción de niños que andan, pedalean y usan el transporte público. El análisis de los datos de la Investigación de Movilidad de 2012 indica no sólo la continuidad, sino la aceleración en el aumento del uso transporte motorizado privado en desplazamientos de niños entre 6 y 11 años hacia la escuela. El efecto de iniciativas en curso sobre esta tendencia sólo será debidamente comprendido con el debido monitoreo de los desplazamientos cotidianos y la evaluación del impacto de esas acciones sobre la salud de la población. Un paquete de políticas y programas dirigidos específicamente para la promoción y protección de la movilidad a pie, de bicicleta o por transporte público de niños, es imprescindible para garantizar el desplazamiento seguro, independiente y activo de niños hacia la escuela en la Región Metropolitana de São Paulo.

Caminata; Transportes; Niño
Recebido em 28/Ago/2015

Versão final reapresentada em 05/Fev/2016 Aprovado em 06/Abr/2016 